\author{
Classification \\ Physics Abstracts \\ $05.00-44.00-60.00-65.00-77.00$
}

\title{
MESURE DE LA DIFFUSIVITÉ DE CÉRAMIQUES FRITTÉES, PAR UNE MÉTHODE FLASH, EN IMPULSION NÉGATIVE, le Chauffage étant aSSuré Par UN JeT De Plasma
}

\author{
M. F. ELCHINGER, C. MARTIN et P. FAUCHAIS
}

Laboratoire de thermodynamique,

Centre céramique (ERA 539) de l'Université de Limoges, 123, rue Albert-Thomas, 87065 Limoges Cedex, France

(Reçu le 23 mars 1978, révisé le 18 septembre 1978, accepté le 20 septembre 1978)

Résumé. - Nous déterminerons par une méthode flash la diffusivité de céramiques frittées. L'échantillon a la forme d'un mince disque de $20 \mathrm{~mm}$ de diamètre et est chauffé sur une de ses faces par le jet d'un générateur à plasma d'arc, un thermocouple appuyé sur l'autre face nous donnant la température de surface de celle-ci.

Nous avons utilisé une méthode d'impulsion négative obtenue par le refroidissement de l'échantillon lorsqu'il cesse d'être chauffé par le jet de plasma dévié, lorsque le régime permanent est établi, pendant un court instant (entre 0,5 et $2 \mathrm{~s}$ ) par un violent jet d'air comprimé qui lui est orthogonal. La diffusivité est alors déterminée en comparant (par la méthode des moindres carrés) la courbe expérimentale de la variation de température correspondante sur la face arrière à la courbe théorique. Pour obtenir cette dernière, l'équation de la diffusion de la chaleur avec des conditions aux limites pour des échantillons de dimensions finies est résolue par la méthode des transformations intégrales qui consiste à déterminer successivement :

- le noyau $K\left(\beta_{m}, r\right)$ de la transformation intégrale considérée,

- la transformation intégrale finie de la température,

- la formule d'inversion de la température.

Le chauffage par jet de plasma nous a permis d'atteindre le régime permanent pour toutes températures en des temps inférieurs à $15 \mathrm{~min}$. Nous pouvons faire varier facilement la température de l'échantillon en jouant sur la nature du gaz plasma ou en modifiant la distance entre la sortie de tuyère et l'échantillon en déplaçant l'échantillon dans le jet.

Nous avons mesuré, pour tester la méthode, la diffusivité de matériaux connus comme le cuivre ou l'aluminium. Les résultats obtenus sont en bon accord avec ceux donnés dans la littérature. Enfin nous avons effectué quelques mesures sur une céramique frittée du type nitrure : l'oxynitrure de silicium.

Abstract. - We determine the diffusivity of sintered ceramic materials. The sample has the shape of a thin disc and is $20 \mathrm{~mm}$ in diameter. We heat it on one of its sides with a plasma jet and a thermocouple is strongly pressed on the other side to give us the temperature of surface of this.

We have used a negative pulse method obtained by the cooling of the sample when it ceases to be heated by the plasma jet deviated, when the permanent running is established, during a short time (between 0.5 and $2 \mathrm{~s}$ ) by a high flow rate compressed air jet that is orthogonal to it. The diffusivity is then determined by comparing (in a last square method) the experimental evolution of temperature on the rear-face to the theoretical curve. To obtain this last, the heat diffusion equation with boundary conditions for samples of finite dimensions are resolved by the integral-transform technique that - consists to determine successively :

- the kernel $K\left(\beta_{m}, r\right)$ of the integral-transform considered,

- the finite integral-transform of temperature,

- the inversion formula of temperature.

The heating by plasma jet allows us to reach the permanent running for any temperatures in times shorter than $15 \mathrm{~min}$. We can change easily the temperature of the sample by changing the nature of plasma gas or by modifying the distance between the nozzle exit and the sample.

We have, as a test, measured the diffusivity of well known materials as copper and aluminium. The results that we have achieved are in accordance with those given in literature. At last, we have measured the diffusivity of a sintered ceramic of the nitrure type : the silicon oxynitride. 
1. Introduction. - Parmi les méthodes employées pour déterminer la diffusivité thermique, celle du signal bref [1] appliquée à des échantillons minces permet d'effectuer des mesures rapides sur des matériaux isolant ou conducteur. Nous pouvons considérer dans cette méthode celle dite de l'impulsion positive et celle dite de l'impulsion négative.

La première consiste à soumettre la face avant d'un petit échantillon à une impulsion d'énergie de courte durée et à observer l'évolution de la température sur la face arrière en fonction du temps. De cette observation, on déduit la diffusivité thermique du matériau étudié.

Dans la seconde, l'échantillon est chauffé en permanence par une source continue (laser, faisceau d'électrons, four à image) qui produit un flux thermique sur une ou sur les deux faces de l'échantillon. Le flux sur une face est alors momentanément interrompu et pendant ce court instant les pertes par rayonnement de l'échantillon vers le milieu environnant réalisent l'impulsion négative.

Les avantages de cette technique, utilisée principalement à hautes températures sont les suivants :

- la température maximale à laquelle l'expérience peut avoir lieu est limitée seulement par le matériau à étudier (degré de vaporisation...) et non par la puissance de la source de chauffage,

- les temps nécessaires pour atteindre la température maximale sont plus brefs que ceux utilisés par les méthodes classiques,

- la technique expérimentale est simplifiée quand la température augmente : en effet, plus la température augmente, plus les pertes thermiques latérales par rayonnement augmentent de telle sorte que des impulsions négatives peuvent être réalisées pendant un temps très court et l'analyse de la mesure est moins sensible aux caractéristiques de l'impulsion entrante. Ceci est plus difficile à réaliser avec des impulsions positives car les pertes thermiques augmentent et on ne peut pratiquement pas introduire une impulsion d'énergie adéquate pour propager une onde thermique observable à travers l'échantillon [2].

Dans ce travail, nous avons repris les travaux de Lincoln et al. [2] et nous avons mis au point une technique de mesure de la diffusivité de céramique en utilisant comme source de chauffage continue un jet de plasma que l'on interrompt pendant un temps très court, pour produire l'impulsion négative, par un puissant jet d'air comprimé orthogonal au jet de plasma.

2. Modélisation théorique de la propagation d'une impulsion négative. - Après avoir indiqué les principales hypothèses de calcul et établi l'équation de la diffusion et les conditions aux limites correspondant à nos hypothèses, nous résolvons le système d'équations exprimé en coordonnées réduites (température, abscisse, temps) par la méthode des transformées de Fourier [3].
2.1 Hypothèses. - Le développement des calculs théoriques relatifs aux impulsions négatives s'appuie sur les hypothèses suivantes :

- l'échantillon est un solide infini (épaisseur faible devant son diamètre) avec des surfaces en $x=0$ et $x=l$ (flux thermique monodimensionnel),

- pour créer l'impulsion négative, à un instant $t$ et pendant un temps très court $t_{\mathrm{p}}$, nous interrompons le flux $\varphi_{0}$ reçu par la face $x=0$,

- un flux uniforme constant $q_{1}$, qui peut être égal à zéro, cas expérimental traité, est absorbé par la surface $x=l$.

- un profil de température stationnaire existe dans l'échantillon avant l'interruption du flux,

- les pertes thermiques superficielles sont essentiellement des pertes radiatives (voir Fig. 1). En effet, selon la loi de Stefan-Boltzmann, la densité de flux thermique exact rayonnant depuis la face avant de l'échantillon $(x=0)$ à la température $T$ et qui cède de la chaleur au milieu environnant (milieu considéré comme un corps noir à la température $T_{\mathrm{s}}$ ) est égale à $\Phi=-\lambda \partial T / \partial x=\varepsilon_{1} \sigma\left(T_{\mathrm{s}}^{4}-T^{4}\right)$ où $\varepsilon_{1}$ est l'émissivité de la face avant de la pastille, $\sigma$ la constante de Stefan-Boltzmann

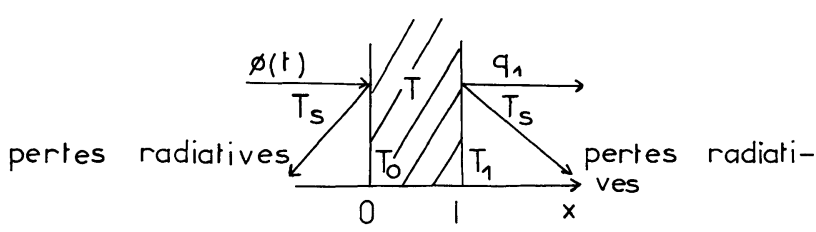

FIG. 1. - Modèle d'un échantillon.

[Sample model (radiative heat losses).]

Nous pouvons faire intervenir une température de référence $T_{0}\left(T_{0}\right.$ étant voisine de $T$ pour un bref refroidissement) et la densité de flux s'écrit alors

$$
\begin{aligned}
\varphi=\varepsilon_{1} \sigma\left[T_{\mathrm{s}}^{4}-\left(T_{0}+\Delta T\right)^{4}\right] \# & \\
& \# \varepsilon_{1} \sigma\left[T_{\mathrm{s}}^{4}-T_{0}^{4}-T_{0}^{3} \Delta T\right]
\end{aligned}
$$

avec $\Delta T=T-T_{0}$, soit finalement

$$
-\lambda \frac{\partial T}{\partial x}=\varepsilon_{1} \sigma\left(T_{\mathrm{s}}^{4}-4 T_{0}^{3} T+3 T_{0}^{4}\right) .
$$

Pour la face $x=l$ à la température $T^{\prime}$, nous pouvons faire le même raisonnement mais en faisant intervenir une température de référence $T_{1}:$ nous obtiendrons :

$$
-\lambda \frac{\partial T}{\partial x}=\varepsilon_{2} \sigma\left(T_{\mathrm{s}}^{4}-4 T_{1}^{3} T^{\prime}+3 T_{1}^{4}\right)
$$

où $\varepsilon_{2}$ est l'émissivité de la face arrière de l'échantillon, $\sigma$ la constante de Stefan-Boltzmann. 
Bien que l'émissivité varie avec la température, nous pouvons supposer que $\varepsilon_{1} \# \varepsilon_{2} \# \varepsilon$ car

$$
T_{1} \# T_{0} \# T^{\prime}
$$

(échantillon mince, impulsion très brève entraînant une faible baisse de température).
2.2 Système A RÉSOUdRE. - Avec ces hypothèses, nous écrivons l'équation de la diffusion thermique et les conditions aux limites sous la forme :

$$
\begin{array}{rlrl}
\frac{\partial^{2} T(x, t)}{\partial x^{2}}=\frac{1}{a} \frac{\partial T(x, t)}{\partial t} & 0<x<l, \quad t>0 \\
-\lambda \frac{\partial T(x, t)}{\partial x}=\varphi_{0}+\varepsilon \sigma\left(T_{\mathrm{s}}^{4}-4 T_{0}{ }^{3} T+3 T_{0}^{4}\right) & x=0, \quad t>0 \\
-\lambda \frac{\partial T(x, t)}{\partial x}=-\varepsilon \sigma\left(T_{\mathrm{s}}^{4}-4 T_{1}^{3} T^{\prime}+3 T_{1}^{4}\right) & x=l, \quad t>0 \\
\varphi_{0}(t) & =q_{0} & t<0 \\
\varphi_{0}(t) & =0 & 0<t<t_{\mathrm{p}} \\
\varphi_{0}(t) & =q_{0} & t>t_{\mathrm{p}} .
\end{array}
$$

En introduisant les variables sans dimension de position $\xi$, de temps $\tau$ et de température $\theta$

$$
\begin{aligned}
\xi & =x / l \\
\tau & =a t / l^{2} \\
\theta & =\left(T-T_{\mathrm{s}}\right) \lambda / 1 q_{0} .
\end{aligned}
$$

Nous avons à résoudre le système ci-dessous :

$$
\begin{aligned}
& \frac{\partial^{2} \theta(\xi, \tau)}{\partial \xi^{2}}=\frac{\partial \theta(\xi, \tau)}{\partial \tau} \quad 0<\xi<l, \tau>0 \\
& \frac{\partial \theta(\xi, \tau)}{\partial \xi}=-F_{0}(\tau)+H_{0} \theta(\xi, \tau)-G_{0} \quad \xi=0, \quad \tau>0 \\
& \frac{\partial \theta(\xi, \tau)}{\theta \xi}=-H_{1} \theta(\xi, \tau)+G_{1} \quad \xi=l, \tau>0 \\
& F_{0}(\tau)=\varphi_{0} / q_{0}=1 \quad \tau<0 \\
& F_{0}(\tau)=0 \quad 0<\tau<\tau_{\mathrm{p}} \\
& F_{0}(\tau)=\varphi_{0} / q_{0}=1 \cdot \quad \tau>\tau_{\mathrm{p}}
\end{aligned}
$$

où

$$
\begin{aligned}
H_{0} & =4 \varepsilon \sigma T_{0}^{3} 1 / \lambda \\
H_{1} & =4 \varepsilon \sigma T_{1}^{3} 1 / \lambda \\
G_{0} & =\varepsilon \sigma\left(T_{\mathrm{s}}^{4}-4 T_{0}^{3} T_{\mathrm{s}}+3 T_{0}^{4}\right) q_{0} \\
G_{1} & =\varepsilon \sigma\left(T_{\mathrm{s}}^{4}-4 T_{1}^{3} T_{\mathrm{s}}+3 T_{1}^{4}\right) q_{0} \\
F_{0} & =\varphi_{0} / q_{0}
\end{aligned}
$$

$F_{0}(\tau)$ définit une impulsion rectangulaire négative dans le flux incident arrivant sur la face avant $(\xi=0)$.

2.3 MÉTHOde DeS TRANSFORMÉES DE FOURIER. Les trois premières étapes du calcul sont classiques [3] (choix de la solution sous forme de variables séparées, détermination de la fonction temporelle et de la fonction spatiale, détermination des racines $\beta_{m}$ de l'équation transcendante suivante :

$$
\operatorname{tg} \beta_{m}=\beta_{m}\left(H_{0}+H_{1}\right) /\left(\beta_{m}^{2}-H_{0} H_{1}\right) .
$$

Nous développerons plus particulièrement les deux dernières étapes qui sont respectivement :

- la détermination du noyau $K\left(\beta_{m}, \xi\right)$ de la transformation intégrale considérée,

- la détermination de la solution- $\theta(\xi, \tau)$ c'est-àdire la température dans l'échantillon à un instant en un point $\xi$.

2.3.1 Détermination du «noyau» $K\left(\beta_{m}, \xi\right)$ de la transformation intégrale considérée. - Nous choi- 
sissons les fonctions propres $\Psi\left(\beta_{m}, \xi\right)$ sous la forme :

$$
\Psi\left(\beta_{m}, \xi\right)=\cos \beta_{m} \xi+\frac{H_{0}}{\beta_{m}} \sin \beta_{m} \xi .
$$

La norme est donnée par :

$$
N=\int_{0}^{1} \Psi^{2}\left(\beta_{m}, \xi\right) \mathrm{d} \xi
$$

Cette intégrale évaluée par Carslaw et Jaeger [4] s'écrit :

$$
N=\frac{1}{2}\left[\frac{\beta_{m}^{2}+H_{0}^{2}}{\beta_{m}^{2}}\left(1+\frac{H_{1}}{\beta_{m}^{2}+H_{1}^{2}}\right)+\frac{H_{0}}{\beta_{m}^{2}}\right] .
$$

Le noyau $K\left(\beta_{m}, \xi\right.$ ) (qui exprime les fonctions aux valeurs propres normalisées) se définit alors comme suit :

$K\left(\beta_{m}, \xi\right)=\frac{\Psi_{m}(\xi)}{\sqrt{N}}=$

$$
=\frac{\sqrt{2}\left[\beta_{m} \cos \beta_{m} \xi+H_{0} \sin \beta_{m} \xi\right]}{\left[\left(\beta_{m}^{2}+H_{0}^{2}\right)\left(1+\frac{H_{1}}{\beta_{m}^{2}+H_{1}^{2}}\right)+\frac{H_{0}}{\beta_{m}^{2}}\right]^{1 / 2}} .
$$

2.3.2 Détermination de la transformation intégrale finie de la température. - Nous transformons l'équation (10) à l'aide de la définition donnée par Ozisik [3]

$$
\int_{0}^{1} K\left(\beta_{m}, \xi\right) \frac{\partial^{2} \theta(\xi, \tau)}{\partial \xi^{2}} \mathrm{~d} \xi=\int_{0}^{1} K\left(\beta_{m}, \xi\right) \frac{\partial \theta(\xi, \tau)}{\partial \tau} \mathrm{d} \xi
$$

qui se met sous la forme :

$$
\int_{0}^{1} K\left(\beta_{m}, \xi\right) \cdot \frac{\partial^{2} \theta(\xi, \tau)}{\partial \xi^{2}} \mathrm{~d} \xi=\frac{\mathrm{d} \bar{\theta}\left(\beta_{m}, \tau\right)}{\mathrm{d} \tau}
$$

Le terme du membre de gauche de l'équation précédente est obtenu en utilisant le théorème de Green [3]. Nous obtenons alors l'équation différentielle linéaire suivante du $1^{\text {er }}$ ordre pour la transformation intégrale de la température :

avec

$$
\frac{\mathrm{d} \theta\left(\beta_{m}, \tau\right)}{\mathrm{d} \tau}+\beta_{m}^{2} \theta\left(\beta_{m}, \tau\right)=A\left(\beta_{m}, \tau\right)
$$

$$
A\left(\beta_{m}, \tau\right)=\frac{\sqrt{ } 2\left(\beta_{m} \cos \beta_{m}+H_{0} \sin \beta_{m}\right) G_{1}+\left(G_{0}+F_{0}(\tau)\right) \beta_{m}}{\left[\left(\beta_{m}^{2}+H_{0}^{2}\right)\left(1+\frac{H_{1}}{\beta_{m}^{2}+H_{1}^{2}}\right)+H_{0}\right]^{1 / 2}}
$$

Pour résoudre cette équation différentielle du $1^{\mathrm{er}}$ ordre linéaire, nous utilisons les conditions initiales définies précédemment (\$2.2, équations (4), (5), (6)).

Nous obtenons alors pour $\theta\left(\beta_{m}, \tau\right)$ l'expression suivante [5] :

$$
\bar{\theta}\left(\beta_{m}, \tau\right)=\frac{\sqrt{2}}{\beta_{m}\left[\left(\beta_{m}^{2}+H_{0}^{2}\right)\left(1+\frac{H_{1}}{\beta_{m}^{2}+H_{1}^{2}}\right)+H_{0}\right]^{1 / 2}} \times\left[\frac{G_{1}\left(\beta_{m} \cos \beta_{m}+H_{0} \sin \beta_{n}\right)}{\beta_{m}}+G_{0}\right]
$$

où

$$
\begin{array}{lrrr}
\Gamma(\tau)=1 & \text { si } & \tau<0 \\
\Gamma(\tau)=\exp \left(-\beta_{m}^{2} \tau\right) & \text { si } & 0 \leqslant \tau \leqslant \tau_{\rho} \\
\Gamma(\tau)=\exp \left(-\beta_{m}^{2} \tau\right)+1-\exp \left(-\beta_{m}^{2}\left(\tau-\tau_{\rho}\right)\right) & \text { si } & \tau>\tau_{\rho} .
\end{array}
$$

2.3.3 Détermination de la température dans l'échantillon à un instant $\tau$ en un point $\xi$. - La formule d'inversion [3] nous permet de revenir à $\theta(\xi, \tau)$ température dans l'échantillon à un instant $\tau$ en un point $\xi$ soit :

$$
\begin{aligned}
\theta(\xi, \tau) & =\sum_{m=1}^{\infty} K\left(\beta_{m}, \tau\right) \bar{\theta}\left(\beta_{m}, \tau\right) \\
& =\sum_{m=1}^{\infty} \frac{2\left(\beta_{m} \cos \beta_{m} \xi+H_{0} \sin \beta_{m} \xi\right)}{\beta_{m}\left[\left(\beta_{m}^{2}+H_{0}^{2}\right)\left(1+\frac{H_{1}}{\beta_{m}^{2}+H_{1}^{2}}\right)+H_{0}\right]} \times\left[\frac{G_{1}\left(\beta_{m} \cos \beta_{m}+H_{0} \sin \beta_{m}\right)}{\beta_{m}}+G_{0}+\Gamma(\tau)\right]
\end{aligned}
$$

où $\Gamma(\tau)(26)$ a déjà été explicité, $\theta(\xi, \tau)$ dépend donc de quatre paramètres $H_{0}, H_{1}, G_{0}, G_{1}((16)$ à $(19))$ et de l'inconnue $a$. 
3. Détermination de la diffusivité à partir des mesures. - Expérimentalement, nous envoyons uniquement sur la face avant de l'échantillon un flux thermique que nous interrompons momentanément. Nous relevons sur la face opposée au flash la variation en fonction du temps de la température $(T=f(t))$ que nous normalisons

$$
\left(\left(T-T_{\text {min }_{1}}\right) /\left(T_{\max }-T_{\min }\right)=f(t)\right) .
$$

Par ailleurs, pour un échantillon donné à une température donnée avec une durée d'impulsion donnée et en initialisant $a, H_{1}, H_{0}, G_{0}, G_{1}$ nous pouvons calculer la variation en fonction du temps, de la température théorique normalisée en tout point de l'échantillon et notamment sur la face arrière.

Il suffit alors de comparer la courbe expérimentale à la courbe théorique par la méthode des moindres carrés. Si l'écart entre ces deux courbes est trop important, il faut réinitialiser $a, H_{1}, H_{0}, G_{0}, G_{1}$ jusqu'à ce que les courbes se superposent.

Le problème consiste donc à initialiser correctement ces paramètres. Nous exposons successivement la méthode des moindres carrés et celle de l'initialisation.

3.1 PRINCIPE DES MOINDRES CARRÉS [6]. - Supposons les constantes déterminées $\left(a, H_{0}, H_{1}, G_{0}, G_{1}\right.$ donc $m=5$ ), le résidu $y_{i}$ entre la valeur $\theta_{i, N \text {,theorique }}$ $\left(\tau_{i}, a, H_{0}, H_{1}, G_{0}, G_{1}\right)$ et la valeur $\theta_{i, N}$ mesurée est tel que :

$$
\sum_{i=1}^{n} y_{i}^{2}
$$

soit minimale [7] ( $n$ étant le nombre de mesures faites et telles que $m<n$ ).

Partant des valeurs initiales $(a)_{0},\left(H_{0}\right)_{0}, \ldots$ nous posons :

$$
a=(a)_{0}+\chi ; \quad H_{0}=\left(H_{0}\right)_{0}+\beta \cdots
$$

et en négligeant les dérivées partielles d'ordre supérieur, et en posant :

$\left[\frac{\partial \theta_{\text {théorique, } \mathrm{N}}}{\partial(a)_{0}}\right]_{\tau=\tau_{i}}=g_{i}, \quad\left[\frac{\partial \theta_{\text {théorique, } \mathrm{N}}}{\left(H_{0}\right)_{0}}\right]_{\tau=\tau_{i}}=\mu_{i} \cdots$ $\theta_{\text {expérimentale,N }}\left(\tau_{i}\right)-\theta_{\text {théorique,N }}\left(\tau_{i},(a)_{0},\left(H_{0}\right)_{0}, \ldots\right)=\varphi_{i}$

l'équation (23) prend la forme :

$$
\sum_{i=1}^{n}\left(g_{i} \chi+\mu_{i} \beta+\cdots-\varphi_{i}\right)^{2}
$$

on écrit la condition du minimum en égalant à zéro les dérivées partielles de l'expression (25) prises par rapport à $\chi, \beta, \gamma \ldots$ d'où le système :

$$
\begin{aligned}
& \sum_{i=1}^{n}\left(g_{i} \chi+\mu_{i} \beta+\cdots-\varphi_{i}\right) g_{i}=0 \\
& \sum_{i=1}^{n}\left(g_{i} \chi+\mu_{i} \beta+\cdots-\varphi_{i}\right) \mu_{i}=0 .
\end{aligned}
$$

Ces équations normales (30) sont en nombre égal au nombre des inconnues $\chi, \beta \ldots$ qu'elles permettent de déterminer [8]. Le système est alors résolu par la méthode de Gauss [9]. Nous avons supposé les constantes $(a)_{0},\left(H_{0}\right)_{0}, \ldots$ connues, nous exposons maintenant la méthode employée pour les déterminer.

3.2 DÉTERmination Des VAleurs INITIALES $(a)_{0}$, $\left(H_{0}\right)_{0},\left(H_{1}\right)_{0},\left(G_{0}\right)_{0},\left(G_{1}\right)_{0}$. - Nous allons faire certaines hypothèses simplificatrices relatives aux pertes thermiques afin de réduire le nombre des paramètres qui doivent être déterminés à partir de l'expérience.

Nous supposerons que :

- pour un échantillon à très haute température rayonnant vers un milieu froid,

$$
T_{\mathrm{s}} \ll T \quad \text { et } \quad T_{\mathrm{s}} \ll T^{\prime}
$$

où $T, T^{\prime}, T_{\mathrm{s}}$ sont respectivement les températures de l'échantillon en $x=0, x=1$ et $T_{\mathrm{s}}$ celle du milieu environnant,

- pour de petites variations de température, on peut remplacer $T_{0}$ par $\hat{T}_{0}$ (température absolue stationnaire) ou $T_{1}$ par $\hat{T}_{1}$ dans la définition de $G_{0}$, $G_{1}, H_{0}, H_{1}$ et pour un équilibre thermique stationnaire relier $G_{0}$ et $H_{0}$, ou $G_{1}$ et $H_{1}$ à $H^{*}$ que l'on pose égal à :

$$
H^{*}=\varepsilon \sigma \hat{T}_{1}^{3} 1 / \lambda
$$

- rappelons également que la face arrière n'est pas chauffée $\left(F_{1}=0\right)$.

Etant donné que la relation de Stefan-Boltzmann pour des pertes thermiques radiatives à partir de la face arrière $(\xi=1)$ s'écrit $H^{*} \theta_{1}$ (grandeur sans dimension) et la différence de température entre les faces avant et arrière $(\xi=0$ et $\xi=1) \theta_{0}-\theta_{1}$, il s'ensuit que l'équilibre thermique de la face arrière conduit à :

$$
\theta_{0}-\theta_{1}=H^{*} \theta_{1} \quad \text { soit } \frac{\theta_{0}}{\theta_{1}}=1+H^{*}
$$

De fait, nous supposons $\varepsilon$ et $\lambda$ indépendants de la température autour de la température d'équilibre (puisque nous avons de petites variations de température autour de celle-ci) et nous écrivons les relations suivantes :

$$
\begin{aligned}
& \frac{H_{0}}{H_{1}}=\frac{\hat{T}_{0}^{3}}{\hat{T}_{1}^{3}}=\left(\frac{\theta_{0}}{\theta_{1}}\right)^{3} \\
& \frac{G_{0}}{G_{1}} \# \frac{\hat{T}_{0}^{4}}{\hat{T}_{1}^{4}}=\left(\frac{\theta_{0}}{\theta_{1}}\right)^{4}
\end{aligned}
$$

A partir de la définition de $G_{1}$ et de $G_{0}$ et de l'équilibre thermique total nous avons

$$
G_{0}+G_{1}=3
$$


En combinant les équations (16) à (19) aux équations (33) à (36) nous en déduisons que :

$$
\begin{aligned}
& H_{1}=4 H^{*} \\
& H_{0}=4 H^{*}\left(1+H^{*}\right)^{3} \\
& G_{0}=3\left(1+H^{*}\right)^{4} /\left(1+\left(1+H^{*}\right)^{4}\right) \\
& G_{1}=3 /\left(1+\left(1+H^{*}\right)^{4}\right) .
\end{aligned}
$$

Les paramètres ont donc été réduits à $a$ et $H^{*}$ mais nous devons les initialiser. Pour cela, nous utilisons la courbe expérimentale $\theta_{\text {face arriere }}(t)$.

Etant donné qu'il y a deux paramètres à déterminer, nous sommes obligés d'utiliser deux valeurs distinctes de la courbe expérimentale. Ces deux valeurs sont :

- d'une part, le temps (noté $t_{1 / 2} \downarrow$ ) compté à partir $\mathrm{du}$ moment où commence l'impulsion et celui où la température de la face $(\xi=1)$ est égale à la demidifférence entre sa valeur à l'équilibre ( $\theta$ équilibre) et sa valeur minimale $\left(\theta_{\text {minimale }}\right)$,

- d'autre part, le temps (noté $t_{1 / 2} \uparrow$ ) compté à partir du moment où commence l'impulsion et celui où la température de la face arrière est revenue à cette même demi-différence (Fig. 2 et Fig. 3).

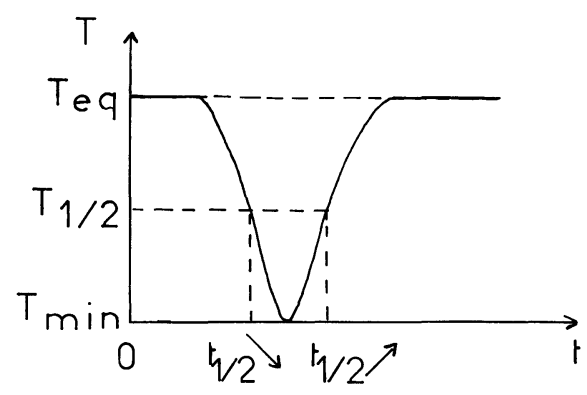

FIG. 2. - Réponse à une impulsion négative (face arrière).

[Temperature variation on the rear face for a negative pulse.]

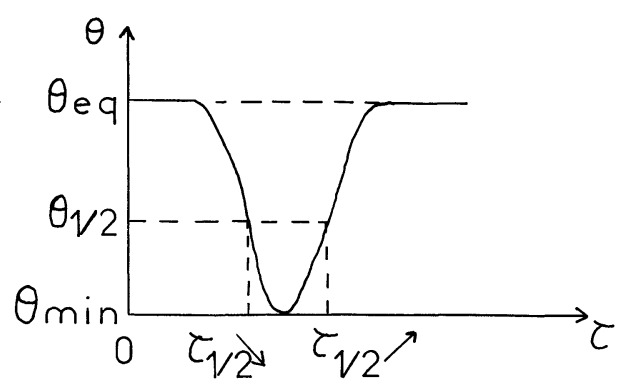

FIG. 3. - Réponse à une impulsion négative (face arrière).

[Reduced temperature variation on the rear face for a negative pulse.]

Nous définissons alors les grandeurs suivantes :

$$
\begin{aligned}
& R_{\mathrm{s}}=\frac{t_{1 / 2} \uparrow}{t_{1 / 2} \downarrow-\frac{1}{2} t_{\rho}}=\frac{\tau_{1 / 2} \uparrow}{\tau_{1 / 2} \downarrow-\frac{1}{2} \tau_{\rho}} \\
& R_{\rho}=\frac{t_{\rho}}{t_{1 / 2} \downarrow}=\frac{\tau_{\rho}}{\tau_{1 / 2} \downarrow} .
\end{aligned}
$$

La seconde égalité, fonction du temps sans dimension, qui figure dans les équations (41) et (42) est écrite pour montrer comment $R_{\mathrm{s}}$ et $R_{\rho}$ sont calculés à partir de l'équation (27). Nous définissons également un nombre réduit de Fourier.

$$
R=\frac{a}{l^{2}}\left(t_{1 / 2} \downarrow-\frac{1}{2} t_{\rho}\right)=\tau_{1 / 2} \downarrow-\frac{1}{2} \tau_{\rho} .
$$

Il nous faut donc tracer maintenant les courbes $R=f\left(R_{\mathrm{s}}\right), R=f\left(R_{\rho}\right), H^{*}=\dot{f}\left(R_{\mathrm{s}}\right)$ c'est-à-dire déterminer les valeurs $\tau_{1 / 2} \downarrow$ et $\tau_{1 / 2} \uparrow$ à partir des courbes théoriques d'évolution de la température $\theta(\xi, \tau)$ calculées pour une valeur minimale et une valeur maximale de la durée de l'impulsion $t_{\mathrm{p}}$, de l'épaisseur de l'échantillon, de la diffusivité $a$ de l'échantillon et de $H^{*}$ c'est-à-dire des températures atteintes et des échantillons considérés (facteur d'émission, conductivité thermique).



FIG. 4. - Organigramme.

[Organigram.] 
Nous avons alors résumé sur les organigrammes des figures 4 et 5 la marche à suivre pour ce calcul. La figure 6 indique par exemple l'allure des courbes théoriques $\theta=f(\tau)$ obtenues pour différentes valeurs de $H^{*}, \tau_{\rho}$ et les figures 7,8 les variations $R=f\left(R_{\mathrm{s}}\right)_{R_{\mathrm{p}}}$ et $H^{*}=f\left(R_{\mathrm{s}}\right)$.

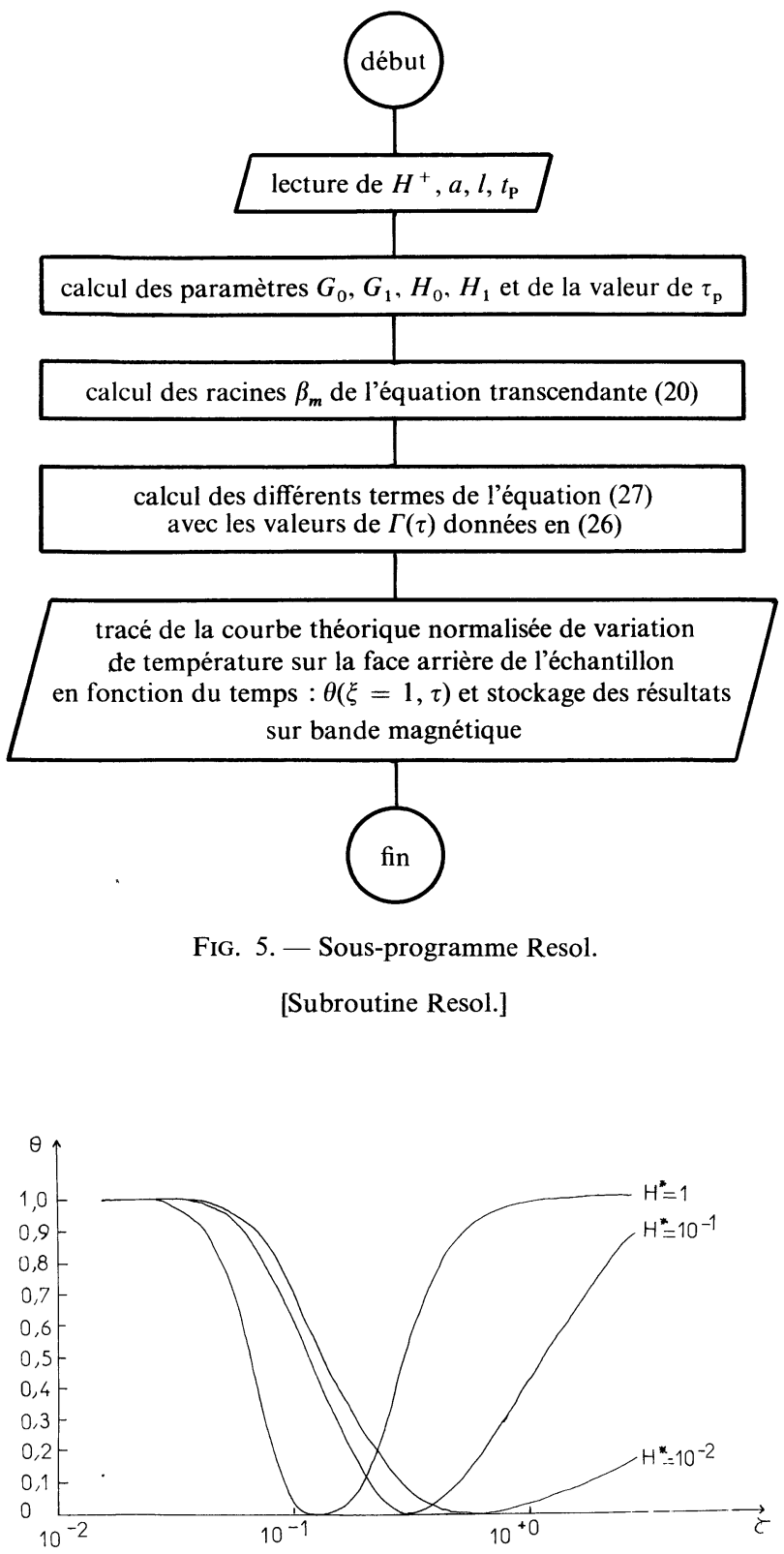

FIG. 6. - Variation de la température sur la face $\xi=1: \tau_{\mathrm{p}}=10^{-2}$.

[Experimental temperature variation on the rear face $(\xi=1$ : $\left.\tau_{\mathrm{p}}=10^{-2}\right)$.]

Expérimentalement, nous connaissons $t_{1 / 2} \downarrow, t_{1 / 2} \uparrow$, $t_{\mathrm{p}}$ donc nous pouvons en déduire $\left(R_{\mathrm{s}}, R_{\mathrm{p}}\right)$. La relation $R=f\left(R_{\mathrm{s}}\right)_{R_{\mathrm{p}}}$ (Fig. 7) nous permet d'obtenir $R$ dont nous déduisons (43) la valeur initiale de la diffusivité $a=R l^{2} /\left(t_{1 / 2} \downarrow-\frac{1}{2} t_{\mathrm{p}}\right)$. Enfin $H^{*}$ (donc $H_{1}, H_{0}, G_{0}$, $\left.G_{1}\right)$ se détermine à partir de la relation $H^{*}=f\left(R_{\mathrm{s}}\right)$ (Fig. 8) [2].

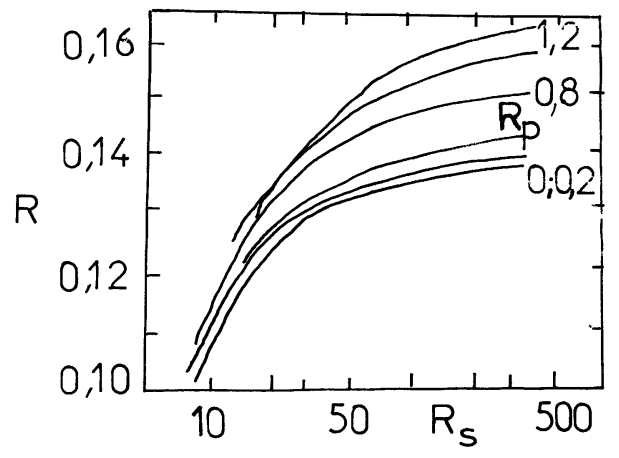

FIG. 7. $-R=f\left(R_{\mathrm{s}}\right) ; R=f\left(R_{\mathrm{p}}\right)$.

$$
\left[R=f\left(R_{\mathrm{s}}\right) ; R=f\left(R_{\mathrm{p}}\right) .\right]
$$

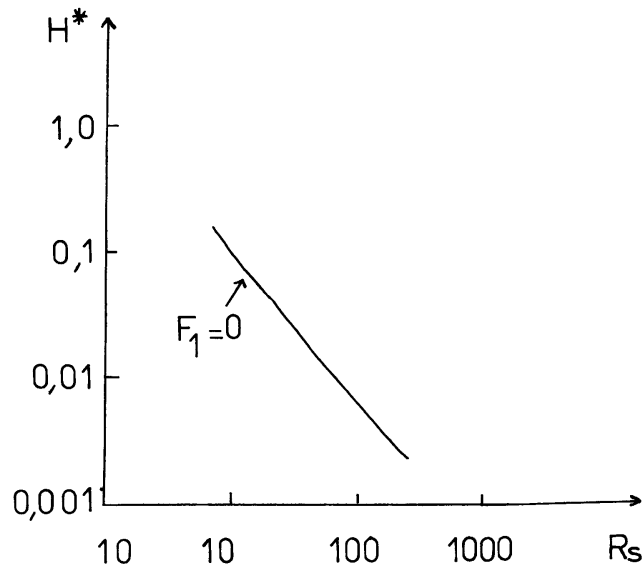

FIG. 8. $-H^{*}=f\left(R_{\mathrm{s}}\right)$.

$$
\left[H^{*}=f\left(R_{\mathrm{s}}\right) \cdot\right]
$$

L'emploi de $\tau_{1 / 2} \downarrow$ n'est pas strictement nécessaire. L'avantage en est que pour des petites impulsions $\left(\tau_{\rho} \ll 1\right) R_{\mathrm{s}}$ et $R$ deviennent insensibles à $\tau_{\rho}$. L'inconvénient est que pour $t_{\mathrm{p}} \# 2 t_{1 / 2} \downarrow$, la technique est inapplicable en raison de la singularité qui existe entre les équations (41) et (42).

\section{Montage expérimental. Résultats expérimentaux.} - 4.1 Montage expérimental. - Le dispositif expérimental (Fig. 9) comporte essentiellement trois parties : l'enceinte de chauffage (1) comprenant le porte-échantillon, l'arrivée d'air comprimé provoquant l'impulsion négative (2) et le dispositif (7) permettant de mesurer la chute de température de la face arrière de l'échantillon.

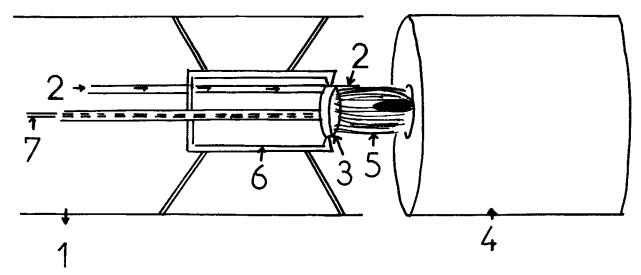

FiG. 9. - Schéma général.

[Schematic arrangement of the whole apparatus.] 
L'échantillon (3) est chauffé en permanence par un générateur de plasma à arc continu stabilisé par écoulement gazeux $([10,12])$. Le jet de plasma est formé d'un dard à très haute température et d'un panache qui englobe l'échantillon, le chauffant ainsi uniformément ce qui diminue les pertes latérales par rayonnement et convection. La température de ce panache pour un gaz diatomique tel que l'azote peut varier de $5000^{\circ} \mathrm{C}$ (si on est assez proche de la tuyère) à $500{ }^{\circ} \mathrm{C}$ (si on est assez loin) alors que la température du dard est de $12000^{\circ} \mathrm{C}$.

L'échantillon est maintenu verticalement et normalement dans le jet (5) à l'aide de trois griffes de tungstène (6) comme indiqué sur la figure 9 . La face avant peut être portée à une température élevée (limitée seulement par la tenue des matériaux et des griffes de maintien). Le tout est enfermé dans une chambre (1) qui limite les fluctuations de température.

Une fois l'équilibre thermique obtenu pour créer l'impulsion négative, nous interrompons momentanément le flux de chaleur qui arrive sur la face $\xi=0$ à l'aide d'un jet d'air comprimé orthogonal au jet de plasma et commandé par une électrovanne dont le temps de réponse est de $10^{-3} \mathrm{~s}$.

La variation de la température sur la face $\xi=1$ est relevée à l'aide d'un thermocoax chromel-alumel de diamètre $1,5 \mathrm{~mm}$ dont la soudure chaude appuie avec une pression constante (à l'aide d'un ressort taré) sur la face arrière de l'échantillon. La disposition du thermocoax sur cette face est un facteur important qui intervient directement sur les résultats. Le signal électrique délivré par le thermocoax est envoyé sur une chaîne d'acquisition de données (Hewlett Packard 3052 A piloté par un calculateur) qui nous donne la courbe de température normalisée en fonction du temps. Pour les températures supérieures

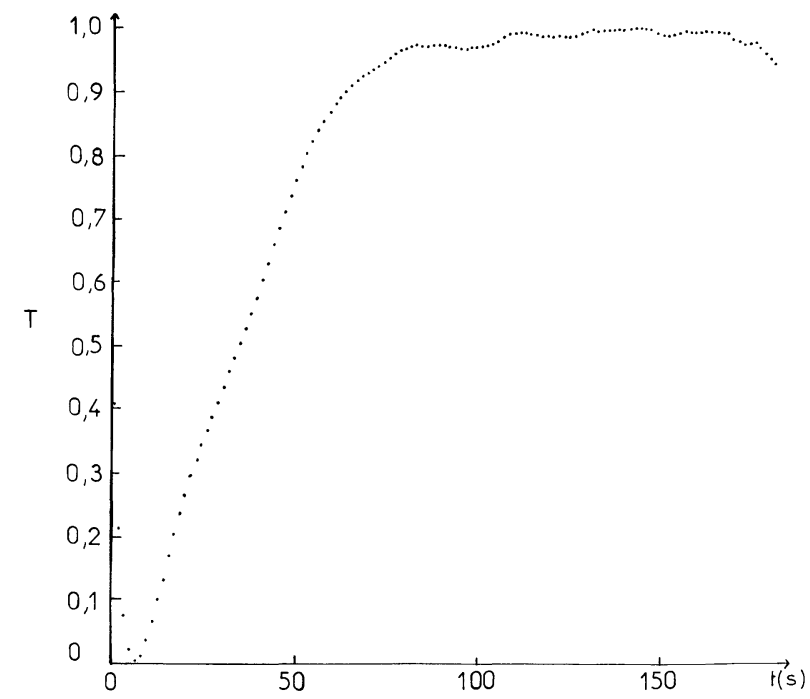

FIG. $10 .-T_{\text {normalisée }}=f(t) \cdot \mathrm{Si}_{2} \mathrm{ON}_{2}: T_{\text {équilibre }}=902^{\circ} \mathrm{C}$, amplitude de l'impulsion $=116^{\circ} \mathrm{C}$.

$\left[T_{\text {normalized }}=f(t) . \mathrm{Si}_{2} \mathrm{ON}_{2}: T_{\text {equilibrium }}=902^{\circ} \mathrm{C}\right.$, magnitude of the pulse $=116^{\circ} \mathrm{C}$.] à $1100 \mathrm{~K}$ la température de la face arrière est également contrôlée par un pyromètre automatique enregistreur à deux couleurs Leybolds.

4.2 Procédure expérimentale. - Nous plaçons l'échantillon dans son support. Nous mettons en fonctionnement le générateur de plasma et la chaîne de mesures. Le programme expérimental stocké sur bandes magnétiques est alors chargé dans la mémoire centrale du calculateur de la chaîne d'acquisition de données. Il contrôle l'équilibre thermique de l'échantillon et une fois celui-ci obtenu (10 à $15 \mathrm{~min}$.), il effectue les opérations suivantes :

- lecture puis moyenne de 1000 températures, - lecture et moyenne des 1000 températures suivantes,
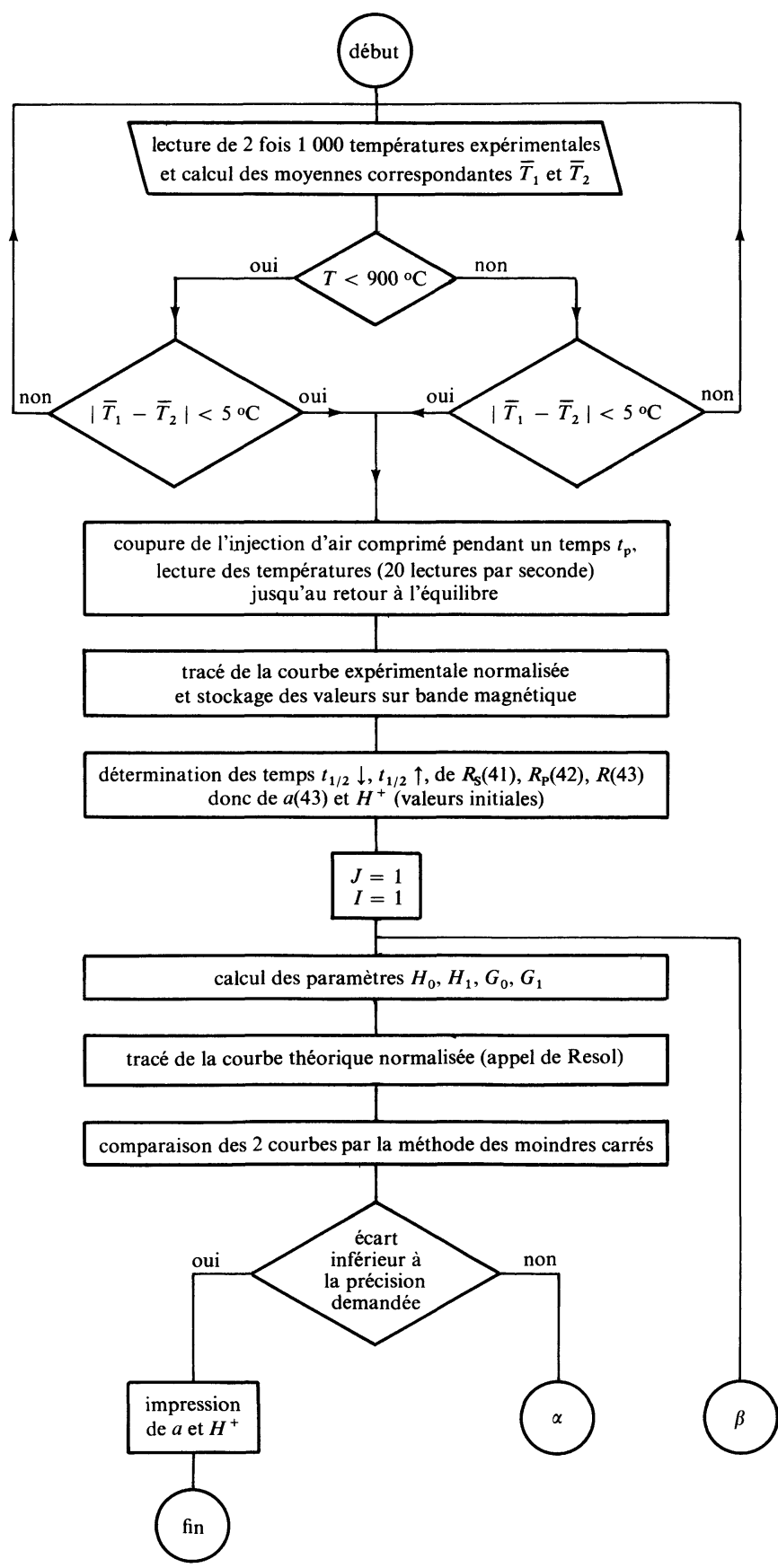
- si l'écart entre ces deux moyennes dépasse $5 \mathrm{~K}$ pour les températures inférieures à $900 \mathrm{~K}$ et $10 \mathrm{~K}$ pour les températures supérieures (ce qui correspond à environ 5 à $10 \%$ de la variation de température sur la face arrière due à l'impulsion négative), nouvelle lecture de deux fois 1000 températures; sinon déclenchement de l'injection d'air comprimé pendant un temps $\tau$ de façon à couper le flux thermique imposé par le plasma sur la face avant. Au cours de cette opération, la chaîne relève d'une part la température (à raison de 20 mesures par seconde) jusqu'au moment où cette dernière est revenue à sa valeur initiale, d'autre part le temps qui s'écoule entre l'injection d'air comprimé et le retour à l'équilibre.

- tracé sur l'imprimante de la chaîne de la courbe de température normalisée (cf. par exemple celle de la figure 10),

- détermination de $a$ et $H^{*}$ à partir des valeurs expérimentales $t_{1 / 2} \uparrow$ et $t_{1 / 2} \downarrow$

- calcul de la courbe théorique correspondante,

- comparaison des deux courbes,

- si l'écart est inférieur à la précision demandée, impression de $a$ et $H^{*}$ sinon modification des valeurs de $a$ et $H^{*}$ et à nouveau tracé d'une courbe théorique.

L'organigramme (Fig. 11) résume cet ensemble d'opérations. On remarquera sur cet organigramme que l'on utilise deux compteurs $i$ et $J$ pour faire varier successivement $a$ puis $H^{*}$ afin de faire coïncider la courbe théorique et la courbe expérimentale.

4.3 RÉSUltats EXPÉRIMENTAUX. - Nous avons testé cette méthode sur un échantillon de cuivre $(\varnothing=20 \mathrm{~mm}, 1=1,5 \mathrm{~mm})$ et un échantillon d'aluminium ayant les mêmes dimensions et dont les caractéristiques figurent dans le tableau I. Les résultats

\section{TABLEAU I}

Caractéristiques du cuivre et de l'aluminium

Aluminium : $99 \%, \mathrm{Al}:$ données théoriques (Raznjevic)

$\begin{array}{ccccc}t\left({ }^{\circ} \mathrm{C}\right) & \rho\left(\mathrm{kg} / \mathrm{m}^{3}\right) & \lambda(\mathrm{W} / \mathrm{m} \mathrm{K}) & c(\mathrm{~W} . \mathrm{s} / \mathrm{kg} \mathrm{K}) & a\left(\mathrm{~m}^{2} / \mathrm{s}\right) \\ - & - & - & - & - \\ 100 & 2700 & 207 & 937 & 8,17 \times 10^{-5} \\ 300 & 2700 & 222 & 1009 & 8,15 \times 10^{-5}\end{array}$

Cuivre commercial : pur $99,98 \%$

$\mathrm{Cu}$ : données théoriques (Raznjevic)

$\begin{array}{ccccc}t\left({ }^{\circ} \mathrm{C}\right) & \rho\left(\mathrm{kg} / \mathrm{m}^{3}\right) & \lambda(\mathrm{W} / \mathrm{m} \mathrm{K}) & c(\mathrm{~W} . \mathrm{s} / \mathrm{kg} \mathrm{K}) & a\left(\mathrm{~m}^{2} / \mathrm{s}\right) \\ - & - & - & - & - \\ 100 & 8930 & 379 & 396 & 10,70 \times 10^{-5} \\ 200 & 8930 & 373 & 405 & 10,30 \times 10^{-5} \\ 400 & 8930 & 364 & 427 & 9,54 \times 10^{-5} \\ 600 & 8930 & 353 & 452 & 8,75 \times 10^{-5}\end{array}$

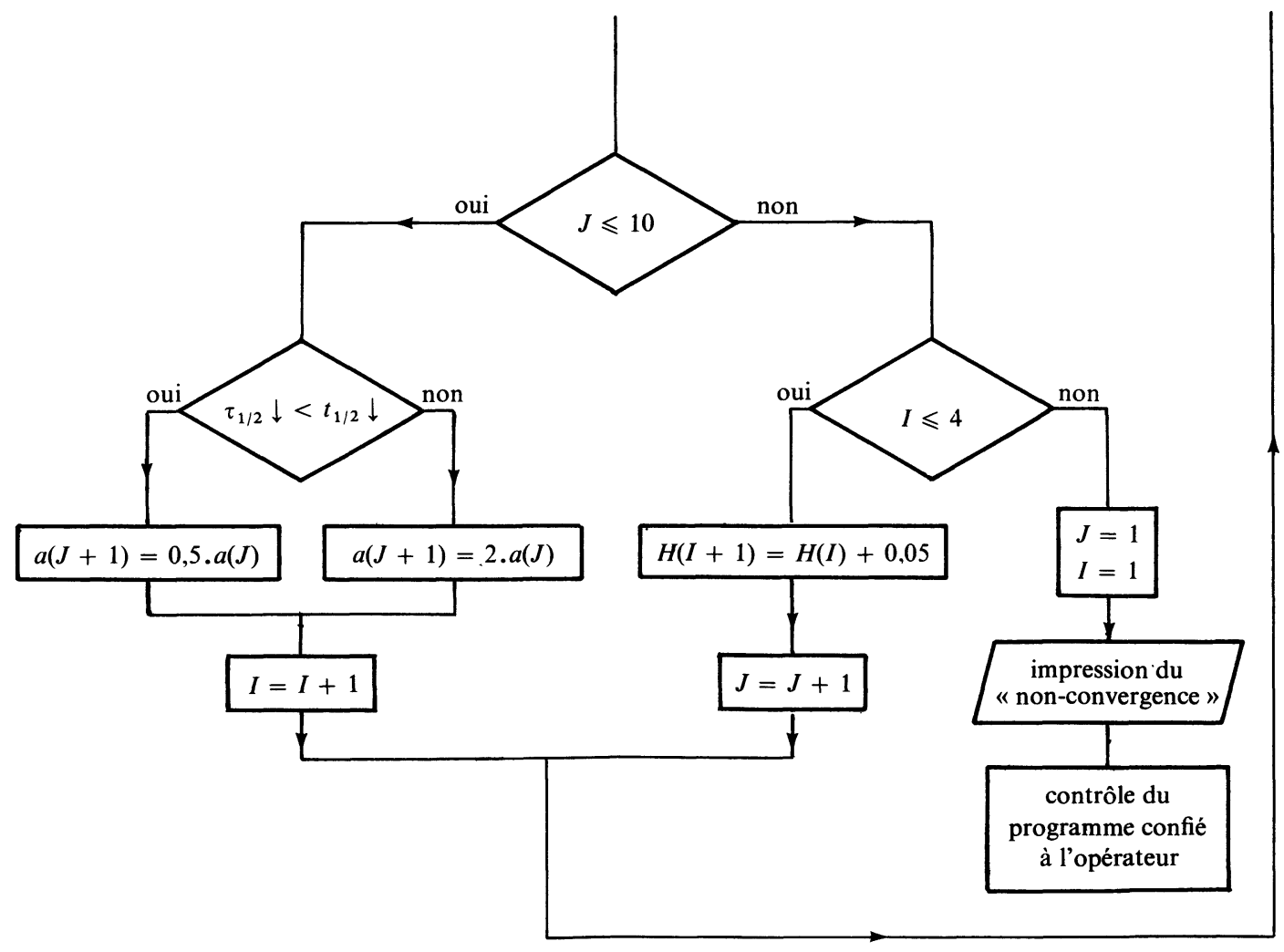

FiG. 11. Organigramme.

$\left(\tau_{1 / 2} \downarrow\right.$ : temps compté à partir de l'instant où commence l'impulsion négative au moment où la température théorique de la face arrière est à mi-chemin entre sa valeur à l'équilibre $\left(\theta_{\text {équilibre }}\right)$ et sa valeur maximale $\left(\theta_{\min }\right) t_{1 / 2} \downarrow$ : temps compté à partir de l'instant où commence l'impulsion négative au moment où la température expérimentale est à mi-chemin entre sa valeur à l'équilibre et sa valeur minimale). 
représentés (Fig. 12) donnent une diffusivité du cuivre variant entre $8,75 \times 10^{-5} \mathrm{~m}^{2} / \mathrm{s}$ et $8,2 \times 10^{-5} \mathrm{~m}^{2} / \mathrm{s}$ pour le domaine de température $500^{\circ} \mathrm{C}$ à $800^{\circ} \mathrm{C}$. C'est l'ordre de grandeur de la diffusivité de ce matériau estimé à partir de la conductivité thermique, de la chaleur spécifique et de la masse volumique indiquée par Raznjevic [13]. Il en est de même pour l'aluminium (Fig. 13).

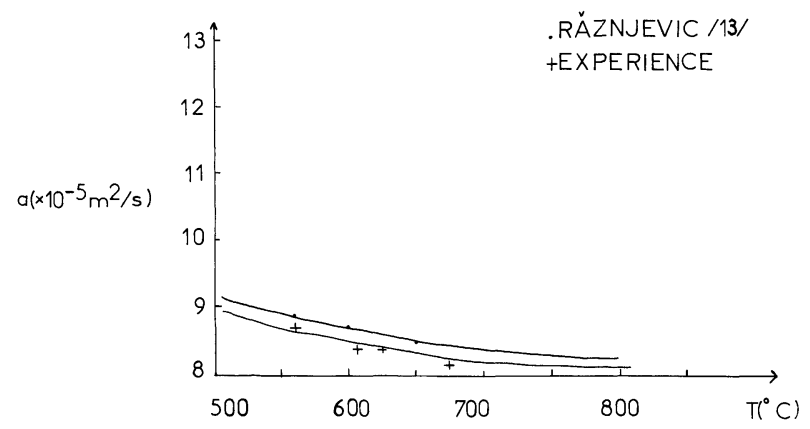

Fig. 12. - Variation de la diffusivité du cuivre en fonction de la température.

[Diffusivity variation of copper versus temperature (experimental result).]

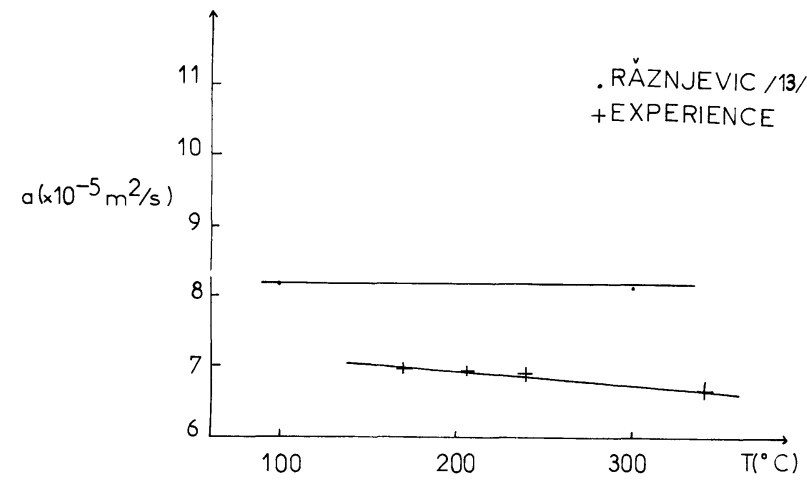

Fig. 13. - Variation de la diffusivité de l'aluminium en fonction de la température.

[Diffusivity variation of aluminium versus temperature (experimental result).]

Nous fondant sur l'expérience ainsi acquise, nous avons testé la méthode sur un échantillon d'oxynitrure de silicium $(\varnothing=20,42 \mathrm{~mm}, 1=1,5 \mathrm{~mm}$, densifié à 2,43 avec un ajout de $\mathrm{MgO}$ et une porosité de 14/100. Les résultats indiqués sur la figure 14 donnent une diffusivité voisine de $2 \times 10^{-6} \mathrm{~m}^{2} / \mathrm{s}$. Cette valeur est du même ordre de grandeur que celle donnée par Washburn M. A. [14] pour des échantillons impurs et de densification plus faible. Il y a donc là toute une série de mesures systématiques à faire sur des échantillons de densification différente et réalisés avec des ajouts divers afin de contrôler ce résultat qui, à première vue, semble indiquer que l'influence de ces paramètres sur la diffusivité est faible.

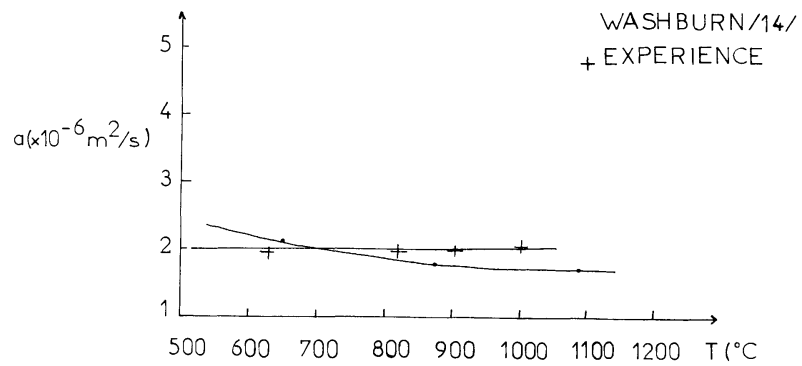

Fig. 14. - Variation de la diffusivité de l'oxynitrure de silicium en fonction de la température.

[Diffusivity variation of silicon oxynitride versus temperature (experimental result).]

4.4 ERreurs. - L'analyse des causes d'erreurs nous amène à faire les remarques suivantes :

— erreurs liées à la mesure du temps $t_{1 / 2} \downarrow, t_{1 / 2} \uparrow$ : la détermination de ces temps nécessite la connaissance de la température d'équilibre et la diminution minimale de la température à partir de la température d'équilibre; elle est donc liée au problème de la mesure d'une température,

- erreurs liées à la mesure des températures : la température enregistrée n'est pas la température réelle de la surface. En effet, cette température est perturbée $\mathrm{du}$ fait des conditions de contact thermocoaxsurface toujours imparfaites (existence d'une résistance thermique de contact). Compte tenu de l'analyse de ce type d'erreurs [15], des appareils dont nous disposons pour faire la mesure, nous pouvons dire que l'incertitude relative sur la mesure est inférieure à $10 \%$. Par ailleurs, pour les températures supérieures à $1100 \mathrm{~K}$ et inférieures à $1500 \mathrm{~K}$ l'écart entre la température mesurée avec le thermocoaxe et celle mesurée avec le pyromètre varie entre 5 et $8 \%$.

Les pertes latérales sont négligeables du fait que l'échantillon est chauffé par le panache du jet de plasma aussi bien sur la face avant que sur ses faces latérales. Pour un échantillon d'acier inoxydable réfractaire dans lequel nous avions disposé 5 thermocouples, au voisinage de sa surface (à $0,1 \mathrm{~mm}$ environ), entre le centre de l'échantillon et sa périphérie, l'écart de température entre le centre et les extrémités est toujours resté inférieur à $2 \%$. Par ailleurs, toujours pour ce même échantillon, nous avons pu constater que les griffes de maintien en tungstène modifiaient de moins de $2 \%$ la distribution des températures de l'échantillon dans un rayon de $3 \mathrm{~mm}$ autour de celles-ci, c'est-à-dire qu'elles ne perturbent pas la zone centrale où est faite la mesure.

Le plasma étant naturellement sujet à des fluctuations de puissance (modification de la longueur de l'arc), nous obtenons alors une variation de la température de quelques degrés autour de la température d'équilibre. Celle légère instabilité peut être augmentée par un courant d'air intempestif (passage de personne, ouvertures de porte ou fenêtre). Nous avons isolé 
l'ensemble (générateur, échantillon, porte-échantillon) dans une enceinte refroidie mais non fermée à ses deux extrémités. Avec celle-ci les fluctuations sont moins prononcées, mais nous pensons pouvoir diminuer toute influence externe parasite avec le nouveau montage en construction où l'ensemble du dispositif est disposé dans une même chambre à atmosphère contrôlée. Par ailleurs, nous développons actuellement un nouveau dispositif de mesure des températures afin d'améliorer la précision de celles-ci.

5. Conclusion. - Dans ce travail nous avons développé les calculs théoriques nécessaires à la mise au point d'une méthode de mesure de la diffusivité thermique de céramiques frittées par impulsion thermique négative, obtenue en interrompant un court instant le chauffage de ces céramiques par un jet de plasma.

Les premiers résultats sont déjà très prometteurs car ils permettent de déterminer la diffusivité avec une précision de l'ordre de $10 / 100$, le chauffage par plasma permettant d'atteindre l'équilibre à haute température en un temps inférieur à $15 \mathrm{~min}$. ce qui est très court par rapport aux autres méthodes de chauffage généralement utilisées dans les méthodes flash décrites dans la littérature.

\section{Bibliographie}

[1] Parker, W. J., Jenkins, R. J., Butler, C. P., Abbott, B. L., J. Appl. Phys. 32 (1979) 1961.

[2] Lincoln, R. C., Donaldson, A. B., Heekman, R. C., J. Appl. Phys. 45 (1974) 2321.

[3] Ozisik, M. N., « Boundary value problems of heat conduction ». International Textbook Company, Scanton, Pennsylvania, 1968.

[4] Carslaw, H. S., Jaeger, J. C., Conduction of heat in solids (Clarendon Press, Oxford), 1959.

[5] Elchinger, M. F., Thèse de 3e cycle, Limoges, 1978

[6] ANGot, A., "Compléments de mathématiques à l'usage des ingénieurs de l'électrotechnique et des télécommunications ». Edition de la Revue d'Optique, Paris, 1965 (5e édition).
[7] Marquardt, D. W., J. Soc. Indust. Appl. Math. 11 (1963) 431.

[8] Paige, C. C., Saunders, M. A., SU, 326, p. 30-29, 22-28, 1973.

[9] GASTINEL, N., Analyse numérique linéaire, p. 78-85.

[10] Fauchais, P., Thèse de Doctorat ès Sciences Physique, 1968.

[11] Bourdin, E., Thèse de 3e cycle, Limoges, 1976.

[12] BoRIE, A., Rapport d'étude du 01/02/76 au 01/08/76, Limoges.

[13] RaZnJeVIC, K., Tables et diagrammes thermodynamiques (Editions Eyrolles), 1970.

[14] Washburn, M. E., Am. Ceram. Soc. Bull. 46 (1967) 667.

[15] Bardon, J. P., "La mesure des températures de surface " Rencontre annuelle de la Société Française des Thermiciens-La Baule 5, 6, 7 mai 1975. 